\title{
Evaluation of Nail Findings in Patients with COVID-19 History and Wood's Lamp Examination
}

\author{
Irem Yanatma ${ }^{\mathrm{a}}$ Hulya Cenk ${ }^{\mathrm{b}}$

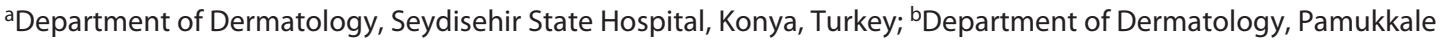 \\ University, Denizli, Turkey
}

\section{Keywords}

Favipiravir · Splinter hemorrhage · Leukonychia •

Fluorescence

\begin{abstract}
Introduction: Various skin findings due to coronavirus have been identified. There are a few case reports on nail findings after coronavirus (COVID-19) infection. We aimed to document the nail findings of the COVID-19 survivors and shed light on the interesting luminescence seen under the Wood's light. Methods: One hundred and seventy-four patients diagnosed with COVID-19 infection in the last 100 days were grouped in terms of the agents used in the treatment. Fiftyseven volunteers without a history of infection were included. Results: Patients treated with favipiravir had a significantly higher positivity of luminescence ( $p$ : 0.0001). The most common nail findings in patients were splinter hemorrhage (13\%), followed by leukonychia (12\%) and longitudinal ridges (7.9\%). Discussion/Conclusions: The luminescence may be seen due to the accumulation of favipiravir or its excipients (titanium dioxide and yellow ferric oxide) on the nails. Wood's lamp examination of the plasma taken from a patient after favipiravir's first dose revealed the same luminescence as we saw on the nails. Accordingly, this accumulation may be seen in the vital organs. Although our
\end{abstract}

knowledge about the virus increases day by day, the potentially hazardous effects of the virus and long-term complications of the treatment options are still being investigated.

(c) 2021 S. Karger AG, Basel

\section{Introduction}

The coronavirus disease (COVID-19 infection) has emerged as a disease in which respiratory system findings such as fever, cough, and shortness of breath are at the forefront. However, over time, it has been shown to affect the gastrointestinal, cardiovascular, and neurological systems. Cutaneous findings associated with the coronavirus have been defined and classified as urticarial, maculopapular, vesicular, petechial rashes, pernio-like acral lesions, and livedo racemosa [1]. Yet, there is no literature knowledge on nail findings of the disease excluding few case reports [2-4].

Favipiravir is an antiviral agent mainly used for influenza treatment as an RNA polymerase inhibitor. It is recommended to be used as orally 1,600 mg, twice daily, on the first day followed by $600 \mathrm{mg}$ twice daily. It has been used to reduce the viral load in the treatment of coronavirus since March 2020 in Turkey [5, 6]. Although hydroxychloroquine (HCQ) was the most commonly used 
Table 1. Sociodemographic data of the patients and nail findings of the patients

\begin{tabular}{|c|c|c|c|c|c|c|}
\hline & favipiravir & $\mathrm{HCQ}$ & $\begin{array}{l}\text { favipiravir plus } \\
\mathrm{HCQ}\end{array}$ & no treatment & healthy & Total \\
\hline \multicolumn{7}{|l|}{ Gender, $n(\%)$} \\
\hline Female & $48(66.7)$ & $15(93.7)$ & $39(69.6)$ & $20(66.7)$ & $35(61.4)$ & $157(67.9)$ \\
\hline Male & $24(33.3)$ & $1(6.3)$ & $17(30.4)$ & $10(33.3)$ & $22(38.6)$ & $74(32.1)$ \\
\hline \multicolumn{7}{|l|}{ Wood's lamp finding, $n(\%)$} \\
\hline Positive & 59 (81.9) & $0(0)$ & 47 (83.9) & $0(0)$ & $0(0)$ & $106(45.9)$ \\
\hline Negative & $13(18.1)$ & $16(100)$ & $9(16.1)$ & $30(100)$ & $57(100)$ & $125(54.1)$ \\
\hline \multicolumn{7}{|l|}{ Systemic disease, $n(\%)$} \\
\hline Positive & 28 (38.9) & $4(25)$ & $22(39.3)$ & $9(30)$ & $13(22.8)$ & $76(32.9)$ \\
\hline Negative & $44(61.1)$ & $12(75)$ & $34(60.7)$ & $21(70)$ & $44(77.2)$ & $155(67.1)$ \\
\hline
\end{tabular}

$\mathrm{HCQ}$, hydroxychloroquine.

drug at the beginning of the pandemic in our country, favipiravir has now been replaced. It has been used with or without HCQ in consistency with the COVID-19 treatment protocol released by the Turkey Ministry of Health [7]. Favipiravir is considered a drug with a low side effect profile [8]. Case reports detecting luminescence under Wood's light in nails after COVID-19 infection were published. The authors associated this finding with the use of favipiravir $[9,10]$. We aimed to document the nail findings of the COVID-19 survivors and shed light on the interesting luminescence.

\section{Materials and Methods}

An ethical approval for the study has been obtained from the local institutional ethics committee and the Ministry of Health Scientific Research Platform. The study was conducted as a multicentric cross-sectional study. Patients were recruited between October and December 2020. Considering the approximate time for the nail growth, patients within the first 100 days of the infection were selected for the study. The study included 174 patients with a history of COVID-19 infection and 57 volunteers without a history of infection. The patients were grouped in terms of the agents used in the treatment, as laid down in the protocol released by the Turkey Ministry of Health [7]. Patients received only favipiravir, only HCQ, favipiravir plus HCQ treatments, and no treatment divided into different groups along with the healthy control group. $\mathrm{Pa}$ tients with current nail infections, a history of chemotherapy, and dermatologic disorders affecting nails were not included in the study.

Nail Findings in Patients with COVID-19
Fingernails were selected for the examination to reduce the risk of viral contamination and shorten the duration of the examination within the scope of pandemic measures. Routine examination findings of the nails and Wood's lamp examination results were recorded after obtaining informed consent. Patients with significant luminescence were considered positive. The nail plate was divided into 3 sections (lunula, middle portion, and distal portion) according to the distance of the luminescence to the nail matrix. Sociodemographic data of the patients including age, gender, and comorbidities were noted.

Data were summarized by median (min-max) and number (percentage). Compliance with normal distribution, KolmogorovSmirnov and Shapiro-Wilk tests were used. Yates in statistical analysis corrected and Pearson $\chi^{2}$ and Kruskal-Wallis tests were used where appropriate. Paired comparisons after Kruskal-Wallis were made using the Bonferroni test. $p<0.05$ value was considered statistically significant. IBM SPSS Statistics 25.0 program was used for the analyses.

\section{Results}

The age and gender distributions of the groups are shown in Table 1. Seventy-two patients had received only favipiravir, 16 of them had received only HCQ, and 56 of them had received favipiravir plus HCQ treatments. Also, 30 patients had not received any treatment since some of them were asymptomatic or some of them had refused the treatment. The volunteers without a history of infection consisted of 57 patients. The average time lapse between the first diagnosis and nail examination was 42 days $(7-100$ 

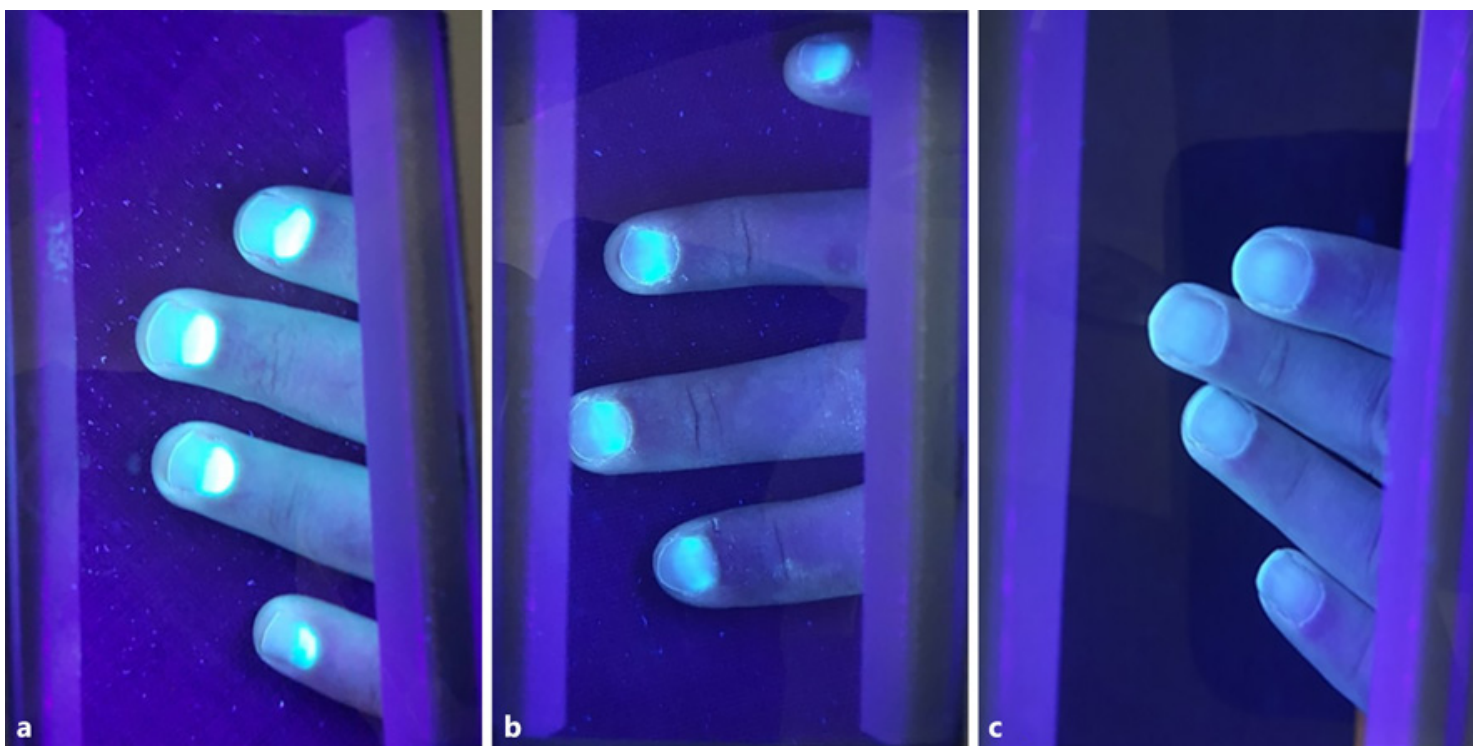

Fig. 1. A patient 34 days after infection who received favipiravir (a). A patient 56 days after infection who received favipiravir (b). A patient 44 days after infection who received hydroxychloroquine (c).

Table 2. Nail findings in the volunteers

\begin{tabular}{|c|c|c|c|c|c|c|}
\hline & \multicolumn{5}{|l|}{ Groups } & \multirow[t]{2}{*}{ Total } \\
\hline & favipiravir & $\mathrm{HCQ}$ & $\begin{array}{l}\text { favipiravir } \\
\text { plus HCQ }\end{array}$ & $\begin{array}{l}\text { no } \\
\text { treatment }\end{array}$ & healthy & \\
\hline None, $n(\%)$ & $41(56.9)$ & $8(50)$ & $36(64.3)$ & $24(80)$ & $41(71.9)$ & $150(64.9)$ \\
\hline Leukonychia, $n$ (\%) & $11(15.3)$ & $2(12.5)$ & $6(10.7)$ & $3(10)$ & $12(21)$ & $34(14.7)$ \\
\hline Splinter hemorrhage, $n$ (\%) & $10(13.9)$ & $4(25)$ & $8(14.3)$ & $1(3.3)$ & $1(1.75)$ & $24(10.4)$ \\
\hline Onychomadesis, $n$ (\%) & 0 & $1(6.25)$ & 0 & 0 & 0 & $1(0.4)$ \\
\hline Onycholysis, $n$ (\%) & $2(2.8)$ & 0 & 0 & 0 & $1(1.75)$ & $3(1.3)$ \\
\hline Pitting, $n$ (\%) & $2(2.8)$ & 0 & 0 & $1(3.3)$ & $2(3.51)$ & $5(2.2)$ \\
\hline Longitudinal ridges, $n$ (\%) & $6(8.3)$ & $1(6.25)$ & $6(10.7)$ & $1(3.3)$ & 0 & $14(6.1)$ \\
\hline Total & 72 & 16 & 56 & 30 & 57 & 231 \\
\hline
\end{tabular}

$\mathrm{HCQ}$, hydroxychloroquine.

days). Approximately, one-third of the patients (32.9\%) had accompanying diseases such as asthma (7\%), thyroid diseases (6\%), diabetes mellitus (4\%), hypertension (3\%), and cancer (2\%). The sociodemographic data and nail findings of the patients are summarized in Table 1.

Luminescence was observed in $81.9 \%$ and $83.9 \%$ of the groups who received only favipiravir and the combined treatment with favipiravir and HCQ, while it was not seen in any of the patients who did not use favipiravir ( $p: 0.0001$ ). In positive cases, the luminescence on the nail was moving away from the matrix with time (Fig. 1). Me- dian days for the distance of the luminescence were 30 (9-83) for the lunula, 58 (33-92) for the middle portion, and $95(75-100)$ for the distal portion, respectively ( $p: 0.0001$ ). $17.1 \%$ of the patients (a total of 128 patients) who were treated with favipiravir did not show luminescence. The average number of days passed after infection of these patients was 66 days.

The most common nail finding was splinter hemorrhage (13.9\%), followed by leukonychia (12.5\%) and longitudinal ridges $(7.9 \%)$ in patients with COVID-19 history. The nail findings are summarized in Table 2. 

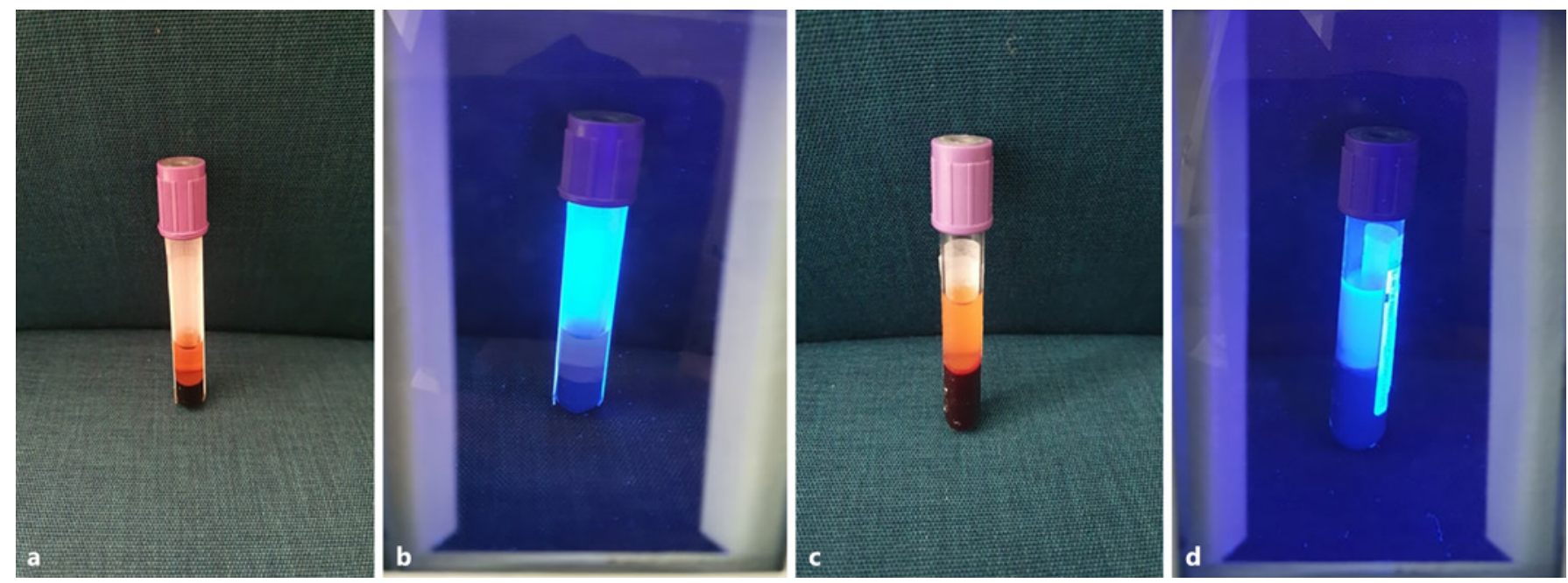

Fig. 2. Blood sample of a patient from the control group after centrifugation (a) and its appearance under the Wood's light (b). Blood sample of a patient treated with favipiravir after centrifugation (c) and the luminescence visible under the Wood's light $(\mathbf{d})$.

Additionally, inspired by the study of Megahed et al. [11], we also looked for the fluorescence in the plasma of a patient. The blood sample has been obtained and centrifuged (4,000 rpm for $15 \mathrm{~min}$ ) $3 \mathrm{~h}$ later of the first intake of favipiravir. And, we have observed that Wood's lamp examination of the plasma revealed the same luminescence as we saw on the nails.

\section{Discussion}

We believe that the luminescence has not been related to a substance that may have been made by coronavirus since the untreated patients with COVID-19 history had not any luminescence in our study. In addition, the absence of luminescence in any patient not receiving favipiravir suggests that this finding is due to favipiravir. We observed that the patients who did not show the luminescence symptoms despite receiving favipiravir were approximately on the 66th day (as a median value) after the treatment. We think that it may be explained by the renewal of the nail plate during the physiological process of nail growth. Complete regrowth of a fingernail may require 4-6 months from the proximal end to the distal edge with an average growth rate of $3 \mathrm{~mm} /$ month (1.8-4.5 $\mathrm{mm} / \mathrm{month})[12,13]$. The growth rate of the nail is influenced by age, gender, seasonality, diseases, and drugs [12].

Nail Findings in Patients with COVID-19
Wood's lamp emits invisible black UV light (320-400 $\mathrm{nm}$ ) which also has a violet light spectrum visible in the dark [14]. Wood's lamp examination is a safe method used for detecting any abnormal [15] fluorescence caused by a photochemical reaction [16] on the skin, hair, nails, teeth, urine, stool, and blood [14]. Wood's light shows different reflections due to different by-products of the microorganisms. Yellowish green, coral red, and vivid green reflections observed under the Wood's light are interpreted as the presence of pyoverdin due to pseudomonas, coproporphyrin due to corynebacteria, pteridine due to microspora species, respectively. Also, porphyrin metabolites accumulated in urine and teeth can be observed by Wood's lamp examination as a pink fluorescence in porphyria [17]. As for drug accumulations on nails, yellow-green and yellow reflections have been defined with mepacrine and tetracycline treatments, respectively [18]. In our study, we have detected a luminescence due to the accumulation of the favipiravir itself or its excipients.

The luminescence may be seen due to excipients (titanium dioxide and yellow ferric oxide) of favipiravir. It has been shown that these metals may accumulate in the internal organs, hair, and nails $[19,20]$. A recent study has shown that plasma concentration of favipiravir and intensity of the luminescence had a linear correlation [11]. Inspired by this study, we looked for the fluorescence in the plasma of a patient. Wood's lamp examination of the plasma revealed the same luminescence as we saw on the nails (Fig. 2). This may be due to the drug's active me-

Skin Appendage Disord 2022;8:136-141 
tabolite or its excipients, but still further studies are needed to uncover the exact cause of the luminescence. We think that it is necessary to be careful in terms of a possible accumulation in the vital organs.

This study is of value by revealing also the nail findings in patients with a history of COVID-19. The most common finding was splinter hemorrhages. They appear as red-brown striae and correspond to hemorrhages or thrombosis of the capillaries $[13,21]$. Trauma or thrombotic diseases such as subacute endocarditis and antiphospholipid antibody syndrome are among the culprits [22]. Although coagulopathy and microvascular vasculitis are associated with COVID-19 infection pathogenesis, more studies are needed to explain the relationship between COVID-19 infection and splinter hemorrhage $[1,23]$. The second most common nail finding in the study was leukonychia. Beau lines and leukonychia associated with COVID-19 infection have been defined in few cases. Beau lines and leukonychia have also been reported to be associated with Kawasaki disease [24]. The authors attribute the similar nail findings in both diseases to the similar inflammation pattern of Kawasaki disease and COVID-19 infection [2,3]. Onychomadesis is a nonspecific finding which can be related to severe infections and drugs. We have observed it in only 1 patient with COVID-19 infection history. Also, a case with onychomadesis associated with COVID-19 infection has been reported in the literature [25]. Besides, red half-moon sign on the lunula and distal orange discoloration have been identified in patients with COVID-19, which were considered to be associated with vascular inflammation [26, 27].

This study aimed to document the nail findings that could be attributed to COVID-19 infection or its treatment. As a limitation, we have a low number of patients in the HCQ-treated group since this agent has become less preferred over time. Although the temporal relation among the disease, treatment, and the findings has been suggestive of a positive correlation, prospective casecontrol studies are required to prove the exact relationship.

\section{Conclusion}

Healthy nails are representatives of good health status, and nails deserve having special attention to find out more about the effect of the diseases and the treatments. A careful examination of the nails may provide some important clues about what is going on inside. With the aid of this study, we wanted to shed light on the luminescence seen in the people with COVID-19 infection history and document the frequent nail findings which may be associated with the infection or the treatment.

\section{Acknowledgment}

A short version of this study was presented verbally at the 6th INDERCOS (Online International Dermatology and Cosmetology Congress) in 2021.

\section{Statement of Ethics}

This study protocol was reviewed and approved by the Pamukkale University Ethics Committee, Approval No. E-60116787-020, and the Ministry of Health Scientific Research Platform. A written informed consent was obtained from participants.

\section{Conflict of Interest Statement}

The authors have no conflicts of interest to declare.

\section{Funding Sources}

This research did not receive any specific grant from funding agencies in the public, commercial, or not-for-profit sectors.

\section{Author Contributions}

Irem Yanatma contributed to conception or design of the work; or the acquisition, analysis, or interpretation of data; drafting the work or revising it critically for important intellectual content; final approval of the version to be published; agreement to be accountable for all aspects of the work. Hulya Cenk contributed to acquisition and interpretation of data, drafting the work or revising it critically for important intellectual content, final approval of the version to be published, and agreement to be accountable for all aspects of the work.

\section{Data Availability Statement}

The data that support the findings of this study are not publicly available because they contain information that could compromise the privacy of research participants but are available from the corresponding author (I.Y.) upon reasonable request. 


\section{References}

1 Gottlieb M, Long B. Dermatologic manifestations and complications of COVID-19. Am J Emerg Med. 2020 Sep;38(9):1715-21.

2 Alobaida S, Lam JM. Beau lines associated with COVID-19. CMAJ. 2020 Sep 8;192(36): E1040.

3 Fernandez-Nieto D, Jimenez-Cauhe J, Ortega-Quijano D, Diaz-Guimaraens B, Dominguez-Santas M, Martinez-Rubio J. Transverse leukonychia (Mees' lines) nail alterations in a COVID-19 patient. Dermatol Ther. 2020 Nov;33(6):e13863.

4 Neri I, Guglielmo A, Virdi A, Gaspari V, Starace $\mathrm{M}$, Piraccini $\mathrm{BM}$. The red half-moon nail sign: a novel manifestation of coronavirus infection. J Eur Acad Dermatol Venereol. 2020 Nov;34(11):e663-5.

5 Cai Q, Yang M, Liu D, Chen J, Shu D, Xia J, et al. Experimental treatment with favipiravir for COVID-19: an open-label Control Study. Engineering. 2020 Oct;6(10):1192-8.

6 Yaghoubi A, Amel Jamehdar S, Movaqar A, Milani N, Soleimanpour S. An effective drug against COVID-19: reality or dream? Expert Rev Respir Med. 2021 Apr;15(4):505-18.

7 https: //covid19.saglik.gov.tr/Eklenti/39061/0/covid-19rehberieriskinhastatedavisipdf.pdf.

8 Türsen Ü, Türsen B, Lotti T. Cutaneous sideeffects of the potential COVID-19 drugs. Dermatol Ther. 2020 Jul;33(4):e13476.

9 Aslan Kayıran M, Cebeci F, Erdemir VA, Aksoy H, Akdeniz N, Gürel MS. Fluorescence of nails and hair on Wood's lamp examination in covid pandemic; undefined effect of favipiravir in humans. Dermatol Ther. 2021 Jan; 34(1):e14740.
10 Kutlu Ö, Yılmaz Ş. Fingernail lunula luminescence in COVID-19 patients: is it a favipiravirrelated reaction or a novel manifestation of coronavirus infection. Photodermatol Photoimmunol Photomed. 2021 Jan 21;37(4):343-4.

11 Megahed SM, Habib AA, Hammad SF, Kamal AH. Experimental design approach for development of spectrofluorimetric method for determination of favipiravir; a potential therapeutic agent against COVID-19 virus: application to spiked human plasma. Spectrochimica Acta A Mol Biomol Spectrosc. 2021 Mar 15;249:119241.

12 Rich P, Kwak HSR. Nail physiology and grooming. Cosmetic dermatology: products and procedures. Wiley-Blackwell; 2010. p. 197-205.

13 Piraccini BM. Nail disorders: a practical guide to diagnosis and management. Springer; 2014.

14 Al Aboud DM, Gossman W. Woods light. StatPearls. Treasure Island (FL): StatPearls Publishing copyright $\odot$ 2021, StatPearls Publishing LLC.; 2021.

15 Klatte JL, van der Beek N, Kemperman PM. 100 years of Wood's lamp revised. J Eur Acad Dermatol Venereol. 2015 May;29(5):842-7.

16 Sato T, Asahina Y, Toshima S, Yaguchi T, Yamazaki K. Usefulness of Wood's lamp for the diagnosis and treatment follow-up of onychomycosis. Med Mycol J. 2020;61(2):17-21.

17 Silverberg JI, Silverberg NB. False "highlighting” with Wood's lamp. Pediatr Dermatol. 2014 Jan-Feb;31(1):109-10.

18 Hendricks AA. Yellow lunulae with fluorescence after tetracycline therapy. Arch Dermatol. 1980 Apr;116(4):438-40.
19 Jani PU, McCarthy DE, Florence AT. Titanium dioxide (rutile) particle uptake from the rat GI tract and translocation to systemic organs after oral administration. Int J Pharm. 1994;105(2):157-68.

20 Abdulrahman F, Akan J, Chellube Z, Waziri $M$. Levels of heavy metals in human hair and nail samples from Maiduguri Metropolis, Borno State, Nigeria. World Environment. 2012;2(4):81-9.

21 Laurent C, Dion J, Régent A. Splinter haemorrhages, splenic infarcts, and pulmonary embolism in granulomatosis with polyangiitis. Vasc Med. 2019 Jun;24(3):263-4.

22 Francès C, Piette JC, Saada V, Papo T, Wechsler B, Chosidow O, et al. Multiple subungual splinter hemorrhages in the antiphospholipid syndrome: a report of five cases and review of the literature. Lupus. 1994 Apr;3(2): 123-8.

23 Al-Samkari H, Karp Leaf RS, Dzik WH, Carlson JCT, Fogerty AE, Waheed A, et al. COVID-19 and coagulation: bleeding and thrombotic manifestations of SARS-CoV-2 infection. Blood. 2020 Jul 23;136(4):489-500.

24 Berard R, Scuccimarri R, Chédeville G. Leukonychia striata in kawasaki disease. J Pediatr. 2008 Jun;152(6):889.

25 Senturk N, Ozdemir H. Onychomadesis following COVID-19 infection: is there a relationship? Dermatol Ther. 2020 Nov;33(6): e14309.

26 Méndez-Flores S, Zaladonis A, Valdes-Rodriguez R. COVID-19 and nail manifestation: be on the lookout for the red half-moon nail sign. Int J Dermatol. 2020;59(11):1414.

27 Tammaro A, Adebanjo GAR, Erasmus HP, Chello C, Pezzuto A, Ramirez-Estrada S, et al. Transverse orange nail lesions following SARS-CoV-2 infection. Dermatol Ther. 2021 Dec 19;34(1):e14688. 